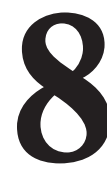

\title{
DETECCIÓN E INTERVENCIÓN EN EL AULA DE LOS TRASTORNOS DE CONDUCTA
}

\author{
(DETECTION AND INTERVENTION IN THE CLASSROOM OF BEHAVIOUR \\ DISORDERS)
}

José Antonio Rabadán Rubio y Ana María Giménez-Gualdo Universidad de Murcia

\section{RESUMEN}

Los trastornos del comportamiento por su preocupante latencia, complejidad y consecuencias negativas en la esfera familiar, académica y social, requieren de una intervención temprana de alta relevancia en los destinatarios formando parte del ámbito de actuación de profesionales del Magisterio, Equipos de Orientación (Pedagogos, Psicopedagogos), Pediatras y Salud Mental. En este sentido, es notable la urgencia de que los profesionales de Educación Primaria y Secundaria den una respuesta acorde a las necesidades del alumnado que presenta tales desórdenes de conducta dentro del aula.

En el presente trabajo, se realiza una concisa revisión teórica de la problemática de los principales trastornos del comportamiento en menores y adolescentes, incluyendo la epidemiología, clínica y factores desencadenantes de los mismos. Tras ello y dando respuesta a la necesidad del profesorado de realizar un acercamiento diagnóstico previo de menores con posibles desórdenes conductuales, presentamos un registro observacional en aras de facilitar esta tarea de detección conducente a una posterior intervención directa con los destinatarios. Por último, se incluyen una serie de pautas educativas recomendables a desarrollar para los tres tipos de trastornos de conducta más comunes tales como el Trastorno por Déficit de Atención e Hiperactividad, Trastorno Negativista Desafiante y Trastorno Disocial. 


\section{ABSTRACT}

Behaviour disorders are a highly relevant area in the treatment and intervention to Teachers, Counselling Teams, Paediatrics and Mental Health Professionals. It is based on the increasing prevalence, complex management and negative consequences in personal, familiar, academic and social fields. For that, it is necessary that teachers in primary and secondary education give an answer in agreement with students' needs who show behaviour disorders inside the classroom.

In this paper, we account for a brief epidemiological and clinical revision of behaviour disorders and their factors in children and teenagers. Because of teachers' need to approach to behaviour disorders, we present an observational tool to make easier the detection of these disorders inside the classroom, which allow them to perform directly with students. Finally, a series of specific educative proposals are included for the three basic behaviour disorders, namely Attention Deficit Hyperactivity Disorder, Oppositional Defiant Disorder and Antisocial Behaviour.

\section{INTRODUCCIÓN}

Nos encontramos en una sociedad en cambio constante en donde la velocidad de dichos cambios ha reducido en gran parte el tiempo de adaptación de las personas. Estos cambios (intrageneracionales, sociales, tecnológicos y comunicativos), crean en muchas ocasiones un desbordamiento de información y la creciente complejidad de que todo lo existente debe ser comprendido por las nuevas generaciones para su integración y desarrollo social. Ante una conducta disruptiva, violenta o agresiva manifestada por un niño o adolescente dentro y fuera del aula, cabe preguntarse, ¿los niños son agresivos porque fueron educados de esta forma o porque nacieron así? Las controversias en torno al origen, desarrollo y mantenimiento de la agresividad han sido objeto de enorme polémica a lo largo de los siglos desde diversos contextos (religión, filosofía, antropología, psicología, etc.), intentando dar una explicación lógica al fenómeno de la violencia que un ser humano ejerce sobre otro (Palomero y Fernández, 2001).

La agresividad puede entenderse como un estado emocional consistente en sentimientos de odio, furia e ira que propicia deseos de dañar a otra persona, animal u objeto (Gerard, 2002). Este estado puede ser consecuencia de situaciones de alta tensión, presión, distorsión cognitiva ante un elemento considerado provocador, la experiencia previa o la conducta de imitación a otros. Es por tanto un concepto amplio y muldimensional que surge de la interrelación tanto de factores orgánicos como ambientales (Alonso y Navazo, 2002). 
En el caso de los niños, cuando nacen sólo están preocupados por sus propias necesidades, lo que les sitúa a ellos mismos como centro de su mundo. No suelen relacionarse con las personas de forma afectiva, sino que las conciben como parte de su mundo, de tal forma que al nacer su actividad es sinónimo de agresión en cuanto que es intrínseca a su proceso de desarrollo. El problema aparece cuando esta agresividad deja de ser un mecanismo de defensa (e incluso de adaptación) para constituirse como una forma de expresión y de relación con el mundo exterior. Comienzan a surgir entonces, conductas, acciones y pensamientos derivados de un desorden comportamental que repercuten de forma negativa tanto en la calidad de vida del propio sujeto como en su contexto inmediato familiar y social.

\section{LOS TRASTORNOS DEL COMPORTAMIENTO EN LA INFANCIA Y LA ADOLESCENCIA}

La conducta se utiliza como medida de la personalidad humana de tal forma que, en gran parte, somos lo que hacemos. Si la conducta comienza a ser desviada, sobrepasa los límites de la normalidad establecidos para vivir o convivir en sociedad y afecta al ámbito personal, familiar y social del individuo, estaremos hablando ya de un trastorno conductual que puede derivar en edades posteriores en trastornos de personalidad, en cuanto patrones conductuales e interacciones interpersonales permanentes en los primeros años de la etapa adulta y con escasa probabilidad de cambio a lo largo de la vida (Oldham, Skodol y Bender, 2007). Los problemas de conducta son para Hill (2003), independientemente de las circunstancias sociales o familiares asociadas, predictores de una personalidad antisocial aunque sólo en el 50\% de los casos, de ahí la importancia y justificación de una intervención precoz.

\subsection{Epidemiología y factores de riesgo en los trastornos conductuales}

Existe una amplia bibliografía sobre la etiología y epidemiología de los trastornos de la conducta en la infancia y la adolescencia que nos proporcionan una información importante sobre el incremento de los índices de prevalencia en los últimos años, los factores que confluyen en su presentación y evolución, los diferentes subtipos, las características diferenciales según la edad de presentación y las diferencias entre sexos (más comunes y severos en niños que en niñas).

Aun teniendo en cuenta las dificultades de investigación, la prevalencia de los desórdenes comportamentales han aumentado preocupantemente en la última década debido a las transformaciones sociales, culturales y edu- 
cativas que estamos experimentando. En términos generales, el porcentaje de trastornos de la conducta diagnosticados en los años 90 no llegaba al 5\% habiéndose triplicado en la actualidad, considerando los trastornos por déficit de atención e hiperactividad (TDAH), trastorno negativista desafiante (TND) y trastorno disocial (TD) (Angulo et al, 2008).

Atendiendo a algunos datos aportados por el Dr. Atienza (n.d.) en relación a la presencia de los trastornos de la conducta más comunes, el TND se sitúa en torno al 4\% obteniendo valores mayores sobre los 8-10 años y durante la adolescencia (13-16 años) siendo más común en chicos que en chicas. El TD se amplía hasta un 9\% en la etapa álgida de la adolescencia y es mayor si se atiende a la variable agresividad y violencia bajo la presencia de una intencionalidad clara de querer causar daño a otra persona (agresivi$\mathrm{dad} /$ violencia hostil) u obtener un beneficio (instrumental).

Si nos centramos en la etiología y/o factores de riesgo de los tres tipos de desórdenes más comunes (TDAH, TND y TD) en la infancia y adolescencia, podemos realizar la siguiente distinción entre: factores genéticos e individuales, familiares y del entorno (Angulo et al, 2008; Domínguez y Pino, 2008; Justicia et al, 2006; San Sebastián, Soutullo y Figueroa, 2010) (Tabla 1). De forma concreta, uno de los factores considerados de mayor relevancia y que correlaciona de forma más positiva con el trastorno conductual y la conducta agresiva manifiesta por el sujeto, es el estilo de crianza de los padres (Raya, Pino y Herruzo, 2009).

\begin{tabular}{|c|c|c|c|}
\hline \multicolumn{4}{|c|}{ FACTORES DE RIESGO } \\
\hline $\begin{array}{l}\text { Genéticos / } \\
\text { Individuales }\end{array}$ & Familiares & Ambientales & Escolares \\
\hline $\begin{array}{l}\text { Escasa habilidades } \\
\text { sociales }\end{array}$ & $\begin{array}{l}\text { Estilos parentales } \\
\text { - Estilo educativo } \\
\text { ineficaz } \\
\text { - Baja supervisión } \\
\text { - Baja estimula- } \\
\text { ción cognitiva } \\
\text { - Escaso apoyo } \\
\text { emocional }\end{array}$ & $\begin{array}{l}\text { Grupo de iguales } \\
\text { con conductas dis- } \\
\text { ruptivas }\end{array}$ & $\begin{array}{l}\text { Escasa atención del } \\
\text { profesorado (énfa- } \\
\text { sis en el rendimien- } \\
\text { to académico y no } \\
\text { en las necesidades } \\
\text { e intereses de los } \\
\text { alumnos) }\end{array}$ \\
\hline $\begin{array}{l}\text { Pobreza en el ma- } \\
\text { nejo de conflictos }\end{array}$ & $\begin{array}{l}\text { Desestructuración } \\
\text { familiar: drogas, } \\
\text { maltrato, conflictos } \\
\text { intrafamiliares }\end{array}$ & $\begin{array}{l}\text { Influencia de los } \\
\text { medios de comuni- } \\
\text { cación como trans- } \\
\text { misores de violencia } \\
\text { permanente (expo- } \\
\text { sición prolongada) }\end{array}$ & $\begin{array}{l}\text { Conductas agresi- } \\
\text { vas en la escuela } \\
\text { (por ej.: bullying) }\end{array}$ \\
\hline
\end{tabular}




\begin{tabular}{|c|c|c|c|}
\hline $\begin{array}{l}\text { TDAH con mayor predo- } \\
\text { minancia en la impulsivi- } \\
\text { dad }\end{array}$ & $\begin{array}{l}\text { Pobreza comunica- } \\
\text { tiva entre los miem- } \\
\text { bros }\end{array}$ & $\begin{array}{l}\text { Acceso libre a } \\
\text { Internet sin su- } \\
\text { pervisión }\end{array}$ & Vandalismo \\
\hline $\begin{array}{l}\text { Dificultades académicas y } \\
\text { de aprendizaje }\end{array}$ & $\begin{array}{l}\text { Estatus socioeconó- } \\
\text { mico }\end{array}$ & $\begin{array}{l}\text { Valores socia- } \\
\text { les: competitivi- } \\
\text { dad, intoleran- } \\
\text { cia, fortaleza, } \\
\text { insensibilidad }\end{array}$ & $\begin{array}{l}\text { Clima de clase } \\
\text { inadecuado }\end{array}$ \\
\hline Acceso a armas & $\begin{array}{l}\text { Trastornos psiquiá- } \\
\text { tricos o de persona- } \\
\text { lidad y de la conduc- } \\
\text { ta en los padres }\end{array}$ & $\begin{array}{l}\text { Afiliación a } \\
\text { ideologías } \\
\text { extremistas }\end{array}$ & $\begin{array}{l}\text { Inteligencia limi- } \\
\text { tada }\end{array}$ \\
\hline $\begin{array}{l}\text { Atribución externa de la } \\
\text { conducta }\end{array}$ & $\begin{array}{l}\text { Depresión post-par- } \\
\text { to }\end{array}$ & $\begin{array}{l}\text { Rechazo a las } \\
\text { normas sociales }\end{array}$ & $\begin{array}{l}\text { Bajo rendimiento } \\
\text { académico y fra- } \\
\text { caso escolar }\end{array}$ \\
\hline $\begin{array}{l}\text { Experiencias de aisla- } \\
\text { miento, rechazo, humilla- } \\
\text { ción }\end{array}$ & $\begin{array}{l}\text { Separación o divor- } \\
\text { cio }\end{array}$ & $\begin{array}{l}\text { Pertenencia a } \\
\text { grupos sociales } \\
\text { deprimidos }\end{array}$ & $\begin{array}{l}\text { Valores culturales } \\
\text { y pedagógicos } \\
\text { (tradicionalista) }\end{array}$ \\
\hline $\begin{array}{l}\text { Ser víctima de acoso (es- } \\
\text { colar o familiar) }\end{array}$ & $\begin{array}{l}\text { Embarazo precoz y } \\
\text { antecedentes de } \\
\text { trastornos de con- } \\
\text { ducta en los padres }\end{array}$ & & $\begin{array}{l}\text { Asimetría entre } \\
\text { profesorado y } \\
\text { alumnado (recha- } \\
\text { zo de las figuras } \\
\text { de autoridad) }\end{array}$ \\
\hline Temperamento fuerte & Adopción & & $\begin{array}{l}\text { Dimensiones de la } \\
\text { escuela (organiza- } \\
\text { ción de espacios, } \\
\text { tiempos...) }\end{array}$ \\
\hline Inflexibilidad & $\begin{array}{l}\text { Complicaciones pe- } \\
\text { rinatales }\end{array}$ & & $\begin{array}{l}\text { Separación/Atri- } \\
\text { bución entre bue- } \\
\text { nos y malos esco- } \\
\text { lares }\end{array}$ \\
\hline $\begin{array}{l}\text { Baja capacidad de frus- } \\
\text { tración }\end{array}$ & $\begin{array}{l}\text { Depresión de la ma- } \\
\text { dre }\end{array}$ & & $\begin{array}{l}\text { Inexistencia de } \\
\text { normas de convi- } \\
\text { vencia }\end{array}$ \\
\hline \multicolumn{4}{|l|}{$\begin{array}{l}\text { Poca capacidad de auto- } \\
\text { control }\end{array}$} \\
\hline \multicolumn{4}{|l|}{$\begin{array}{l}\text { Actitud negativa, desa- } \\
\text { fiante }\end{array}$} \\
\hline Consumo de sustancias & & & \\
\hline
\end{tabular}




\begin{tabular}{|l|l|l|l|}
\hline $\begin{array}{l}\text { Búsqueda constante de } \\
\text { experiencias (peligro- } \\
\text { sas) }\end{array}$ & & & \\
\hline Gemelos monocigóticos & & & \\
\hline $\begin{array}{l}\text { Desajustes en la dopa- } \\
\text { mina, serotonina, cal- } \\
\text { cio, noradrenalina y áci- } \\
\text { do GABA }\end{array}$ & & & \\
\hline Alteraciones cerebrales & & & \\
\hline $\begin{array}{l}\text { Enfermedad crónica: } \\
\text { epilepsia, diabetes }\end{array}$ & & & \\
\hline
\end{tabular}

Tabla 1. Factores de riego durante la infancia y la adolescencia

Abordar la conducta problemática de los niños y jóvenes en el contexto escolar y la familia, es importante para realizar un diagnóstico lo más preciso posible (constrastable y discutible entre los diversos profesionales que se embarcan en el tratamiento del menor) ahondando en las posibles causas de tales desórdenes. Estos factores constituyen un riesgo o catalizador para el desarrollo de ciertas conductas que se alejan o desvían de los parámetros considerados normales (Moreno, 2000). Este conocimiento ayudará sobremanera al diagnóstico de la problemática y guiará los posibles tratamientos a llevar a cabo con el menor.

\subsection{Clínica y formas de presentación}

Por lo general, los docentes de los niveles de educación primaria y secundaria, van a percibir, en los estadios iniciales del desarrollo del niño, la presencia de alteraciones conductuales en el aula. Incluso los propios padres, en los horarios de atención a los mismos, van a manifestar las dificultades para manejar al niño de forma adecuada, el exceso de rabietas o los problemas para relacionarse con sus iguales. El número de horas que comparte el docente con el niño y los comportamientos que muestra en el aula, hacen que el maestro esté en una posición idónea para detectar la presencia de estos trastornos conductuales, lo que justifica nuestro objetivo de presentar el instrumento que más adelante explicaremos.

La sintomatología de los trastornos de comportamiento viene definida en los criterios diagnósticos y estadísticos de las clasificaciones internacio- 
nales más comunes como son: el Manual Diagnóstico y Estadístico de los Trastornos Mentales (DSM-IV TR) (APA, 2002) y la Clasificación Estadística Internacional de enfermedades y otros problemas de salud (CIE-10) (OMS, 2008). Los criterios diagnósticos reflejados en el CIE-10, referidos concretamente a los trastornos conductuales son:

\section{F91 Trastornos Disociales}

- F91.0 Trastorno disocial limitado al contexto familiar

- F91.1 Trastorno disocial en niños no socializados

- F91.2 Trastorno disocial en niños socializados

- F91.3 Trastorno disocial desafiante y oposicionista

- F91.8 Otros trastornos disociales

- F91.9 Trastorno disocial sin especificación

\section{F92 Trastornos Disociales y de las emociones mixtos}

- F92 Trastornos mixtos de la conducta y de las emociones

- F92.0 Trastorno depresivo de la conducta

- F92.8 Otros trastornos mixtos de la conducta y de las emociones.

- F92.9 Trastorno mixto de la conducta y de las emociones, no especificado

Del mismo manual podemos desprender 23 posibles síntomas o conductas manifiestas (Tabla 2) que los niños y adolescentes presentan a lo largo de su desarrollo y trastorno comportamental en los diversos contextos, ayudándonos a facilitar su detección y diagnóstico dentro y fuera del aula. 
1. Presenta accesos de cólera anormalmente frecuentes y violentos, teniendo en cuenta su nivel de desarrollo

2. Discute frecuentemente con los adultos

3. Se opone activamente a las demandas de los adultos o desobedece

4. Realiza frecuentemente, de forma deliberada, cosas que contrarían a los adultos

5. Acusa frecuentemente a otros de ser responsables de sus faltas o de su mala conducta

6. Es frecuentemente susceptible o contrariado por los demás

7. Es frecuentemente rencoroso o vengativo

8. Es frecuentemente malo o vindicativo

9. Miente frecuentemente o no mantiene sus promesas, para obtener objetos o favores o para evitar obligaciones

10. Comienza frecuentemente peleas (no tener en cuenta peleas entre hermanos y hermanas

11. Ha utilizado un arma que puede herir seriamente a otro (por ejemplo un bastón, un ladrillo, una botella rota, un cuchillo, un arma de fuego)

12. Permanece fuera de casa por la noche a pesar de la prohibición de los padres

13. Ha sido físicamente cruel hacia personas

14. Cruel hacia los animales

15. Ha destruido deliberadamente bienes de los demás

16. Ha prendido fuego pudiendo provocar, o para provocar destrozos importantes

17. Robo de objetos de valor, sin enfrentarse a la víctima, en la casa o fuera de la casa

18. Hace novillos con frecuencia, desde la edad de 13 años o antes

19. Se ha fugado al menos dos veces o al menos una vez no ha vuelto hasta el día siguiente, viviendo con sus padres o en acogida familiar (no cuentan las fugas para evitar el castigo)

20. Ha cometido algún delito con enfrentamiento a la víctima

21. Ha obligado a alguien a tener una actividad sexual

22. Molesta frecuentemente a otras personas (hiere, les hace sufrir, intimida..)

23. Ha entrado en la casa violentándola, o en un inmueble o en el coche de otra persona 
Todo lo apuntado nos permite tener una visión bastante completa de cuáles son las conductas más comunes en niños y adolescentes con trastornos del comportamiento, la epidemiología de tales desórdenes y los factores de riesgo condicionantes de su aparición y mantenimiento en el tiempo.

\section{PRINCIPALES MANIFESTACIONES DE LOS TRASTORNOS DE LA CONDUCTA}

En primer lugar cabe hacer mención a que la clasificación que se hace de los principales trastornos del comportamiento, difiere dependiendo de los criterios utilizados (Félix, 2007). Una primera clasificación hace referencia a la presencia (exceso) o ausencia (déficit) de cierta desviación con respecto a lo considerado normal en un determinado contexto. Si se atiende a la dirección o consecuencias del comportamiento, podemos distinguir entre conductas externalizantes como la agresividad o internalizantes como la depresión o ansiedad, elementos existentes en los sujetos con trastornos comportamentales. La tercera clasificación más utilizada es la que atiende a los criterios diagnósticos presentes en el DSMIV-TR y el CIE-10, en donde aparecen como trastornos de la conducta más comunes el TDAH, TND y TD.

\subsection{Trastorno por déficit de Atención e Hiperactividad (TDAH)}

El TDAH, como trastorno psiquiátrico más frecuente y comúnmente diagnosticado durante la etapa escolar (Félix, 2007; San Sebastián, Soutullo y Figueroa, 2010) es un síndrome caracterizado por un desarrollo alterado en los mecanismos reguladores de la atención y la reflexividad (Miranda, Jarque y Soriano, 1999) lo que supone un déficit de atención, en la actividad (hiperactividad) e impulsividad. Aunque es poco común encontrarlo en su estado puro ya que existen altos índices de comorbilidad con otros desórdenes, se puede presentar en mayor porcentaje en cualquiera de sus tres rasgos: predominancia en inatención (TDAH-I/TDA-DA), en hiperactividad-impulsividad (TDAH-HI) o el tipo combinado (TDAH-C) (Angulo et al, 2008).

El TDAH como trastorno más generalizado durante la infancia, supone más de la mitad de las consultas en pediatría y psiquiatría infantil y más del 10\% de los comportamientos disruptivos. Aunque su prevalencia dista en función de los criterios diagnósticos que se utilicen, la población utilizada, el método (Artigas, 2003), edad o fuentes de información, los datos más recientes apuntan al 2-12\% en la población infanto-juvenil y una media entre el 5-8\% (San Sebastián, Soutullo y Figueroa, 2010).

Los rasgos más comunes (Tabla 3) que presentan los niños con TDAH atienden a sus tres principales características de hiperactividad, impulsivi- 
dad e inatención. Estos síntomas son (APA, 2002; Félix, 2007; Jara, 2009; San Sebastián, Soutullo y Figueroa, 2010):

\begin{tabular}{|c|c|c|}
\hline INATENCIÓN & HIPERACTIVIDAD & IMPULSIVIDAD \\
\hline $\begin{array}{l}\text { No presta atención a los } \\
\text { detalles }\end{array}$ & $\begin{array}{l}\text { Se mueve continuamente } \\
\text { de su asiento }\end{array}$ & $\begin{array}{l}\text { Adelanta las respuestas } \\
\text { antes de terminar las pre- } \\
\text { guntas }\end{array}$ \\
\hline $\begin{array}{l}\text { Dificultad para la aten- } \\
\text { ción persistente y mante- } \\
\text { nida }\end{array}$ & $\begin{array}{l}\text { No permanece sentado } \\
\text { cuando así lo requiere la } \\
\text { situación o actividad }\end{array}$ & $\begin{array}{l}\text { Suele interrumpir (inin- } \\
\text { tencionadamente) a las } \\
\text { personas mientras hablan }\end{array}$ \\
\hline $\begin{array}{l}\text { Parece que no escucha } \\
\text { cuando se le habla }\end{array}$ & $\begin{array}{l}\text { Está sobreactivado (co- } \\
\text { rre, salta de forma inade- } \\
\text { cuada) }\end{array}$ & $\begin{array}{l}\text { Es incapaz de esperar sus } \\
\text { turno }\end{array}$ \\
\hline $\begin{array}{l}\text { Dificultad para acabar } \\
\text { tareas empezadas y se- } \\
\text { guir instrucciones u obli- } \\
\text { gaciones }\end{array}$ & $\begin{array}{l}\text { Es ruidoso durante las } \\
\text { actividades }\end{array}$ & $\begin{array}{l}\text { Actúa/Responde de forma } \\
\text { precipitada, sin pensar en } \\
\text { las respuestas o conse- } \\
\text { cuencias derivadas de sus } \\
\text { acciones }\end{array}$ \\
\hline $\begin{array}{l}\text { Se distrae por cualquier } \\
\text { estímulo externo }\end{array}$ & Habla de forma excesiva & \\
\hline Es olvidadizo & $\begin{array}{l}\text { Parece sobrarle energía } \\
\text { ya que no para quieto }\end{array}$ & \\
\hline Es desordenado & $\begin{array}{l}\text { Tiene dificultad para rea- } \\
\text { lizar/participar en activi- } \\
\text { dades tranquilas }\end{array}$ & \\
\hline \multicolumn{3}{|l|}{$\begin{array}{l}\text { Dificultad para organizar } \\
\text { tareas }\end{array}$} \\
\hline \multicolumn{3}{|l|}{ Extravía objetos } \\
\hline $\begin{array}{l}\text { Rehúye realizar tareas } \\
\text { que supongan concentra- } \\
\text { ción y permanencia aten- } \\
\text { cional }\end{array}$ & & \\
\hline
\end{tabular}

Tabla 3. Síntomas presentes en el TDAH 
La evaluación del TDAH como desorden atencional, de sobreactividad e impulsividad, debe ser ante todo multidisciplinar, esto es, provenir de diversas fuentes de información que ayuden a corroborar cada una de los diagnósticos, puesto que en ocasiones se ha tipificado de hiperactivos a niños que en realidad no lo eran. Esta diversidad de fuentes, permitiría acertar más en cuanto a la prevalencia del trastorno ya que «en la mayoría de los estudios se observa una diferencia entre los casos comunicados por los padres y los comunicados por los maestros, con una mayor proporción de casos identificados sólo por los padres y una proporción menor correspondiente al consenso entre ambos» (Peña y Montiel, 2003, 175). Tener en cuenta a los diferentes informantes contribuye a que el diagnóstico y en consecuencia el tratamiento establecido para el menor, sea mucho más acertado y acorde con los síntomas manifiestos y sus verdaderas necesidades (Montiel y Peña, 2001).

\subsection{Trastorno Disocial (TD)}

Los trastornos disociales cabe entenderlos como aquellos patrones conductuales a partir de los cuales el sujeto viola de forma persistente y permanente en el tiempo los derechos básicos de otras personas o transgrede normas sociales adecuadas para su edad (Angulo et al, 2008; APA, 2002; Obrero, 2009).

La prevalencia de este trastorno se sitúa entre el 2 y el 10\% en la población femenina y en porcentajes mayores entre los hombres (6-16\%) (Félix, 2007). Aunque en ocasiones puede confundirse con conductas disruptivas o conflictos puntuales en los centros educativos, es el trastorno que en mayor medida se manifiesta en las aulas.

Según el DSM-IV-TR los criterios diagnósticos o síntomas comunes manifiestos de este trastorno, son los cuatro que recogemos a continuación:

\section{- Agresión a personas y animales}

- El sujeto suele amenazar o intimidar a otras personas (de forma verbal o haciendo uso de algún objeto tal como pistola, bate, ladrillo o cualquier otro que pueda causar daño físico a la víctima).

- Se inicia en peleas.

- Manifiesta una conducta cruel hacia personas o animales.

— Fuerza/ha forzado a alguien a mantener con él relaciones sexuales. 


\section{- Destrucción de la propiedad ajena}

- De forma claramente intencional y deliberada: destruye las propiedades de otras personas haciendo uso, en algunos casos, del fuego para causar el mayor daño posible.

\section{- Robo}

- Comete hurtos en el propio hogar o en la propiedad/es de otras personas enfrentándose y causando daño a la víctima (también puede ocurrir que no haya enfrentamiento).

- Miente para obtener algún beneficio/favor o evitar obligaciones.

\section{- Violación grave de las normas sociales}

- No es capaz de asumir las normas sociales básicas de convivencia, por lo que transgrede las pautas o prohibiciones exigidas por los padres.

- Se escapa del hogar y no regresa a éste durante un tiempo.

- Se abstiene de forma periódica de asistir a clase.

También suele darse en estos niños y adolescentes afectados una falta de empatía y conciencia que les lleva a no ser conscientes y responsabilizarse del daño que pueden causar a las personas con sus actos, como también ausencia de culpa, baja tolerancia a la frustración, impulsividad, acusan de sus actos a otros compañeros o personas, abusan de sustancias y llegan a cometer actos delictivos (Obrero, 2009).

La consideración de trastorno disocial en el individuo exige que al menos tres de estas conductas hayan formado parte del comportamiento cotidiano del niño o adolescente durante el último año o haya puesto de manifiesto uno de los síntomas anteriores en los últimos seis meses.

\subsection{Trastorno Negativista Desafiante (TND)}

Como su propio nombre indica, el TND se define por la presencia recurrente y mantenida en el tiempo de un comportamiento deliberado marcadamente desafiante, desobediente, provocador, no cooperativo, negativo, irritable, en constante mal humor y de oposición generalmente a figuras o personas que representen autoridad especialmente de la familia y la escuela (Angulo et al, 2008; Obrero, 2009; Soler, 2010), distinguiéndose del TD en 
cuanto que no viola los derechos básicos de otras personas.

Estos niños muestran rencor, molestia o resentimiento permanente con todo y todos, son vengativos, poco tolerantes a la frustración, groseros, hacen un mal uso del lenguaje, mienten, tienen problemas académicos y se hacen las víctimas de su situación acusando a otros de su mal comportamiento (Félix, 2007; Obrero, 2009).

Como sucedía en el caso de los dos anteriores trastornos comentados (TDAH y TD), es necesario para ser catalogado de trastorno que tal comportamiento se haya mantenido en el tiempo por lo menos seis meses y que exista un deterioro en la calidad de vida personal, social y académica del sujeto. Un aspecto importante a considerar es que estas conductas manifiestas suelen darse de forma más común en el contexto familiar que en la escuela.

Entre los criterios diagnósticos que especifican los síntomas más comunes de este desorden (DSM-IV-TR, 2002), encontramos además de los ya apuntados que con frecuencia: encoleriza e incurre en pataletas, discute y desafía activamente a los adultos, rechaza cumplir obligaciones impuestas, molesta y suele ser molestado por otros. Para una evaluación positiva del mismo, es necesario que se cumpla el criterio de tiempo y de «no normalidad» en referencia al estadio evolutivo del menor.

Por la naturaleza de este trastorno, se recomienda un tratamiento terapéutico de índole más psicológico o conductual como la psicoterapia individual, terapia familiar, terapia grupal con pares, terapia cognitiva (Soler, 2010), siendo desaconsejado el tratamiento farmacológico, siempre y cuando no existan otros desórdenes asociados a éste (Félix, 2007).

\section{UN EJEMPLO DE INSTRUMENTO PARA DETECTAR LOS TRASTORNOS DE LA CONDUCTA EN EL AULA}

Es un hecho suficientemente conocido la dificultad con que se encuentra la inmensa mayoría de los padres y profesores para lograr identificar los indicadores prematuros que pueden apuntar hacia un trastorno de la conducta; dificultad que se amplía incluso a pediatras, psiquiatras, psicólogos y pedagogos cuando se plantea quién o quiénes son los profesionales que deben participar en el diagnóstico y tratamiento de tales desórdenes. Esta cuestión ha venido teniendo distintas respuestas en función de la orientación sobre la etiología y efectos que tienen los trastornos a que nos referimos, así como las técnicas para tratarlos. Ante esta escasez de recursos (humanos y materiales), pretendemos aportar al profesional de la educación una sencilla 
herramienta para la detección y el diagnóstico primario de estudiantes con trastornos de la conducta.

Las principales dificultades con que se enfrentan padres, docentes y el resto de profesionales en este ámbito de los trastornos conductuales son las referidas al diagnóstico y las dificultades para la prevención y el tratamiento (fata de información en la familia, escasa formación del profesorado, ausencia de conocimiento de estrategias educativas y psicológicas para el tratamiento en el aula y el hogar, escasez de profesionales, etc.).

Para facilitar esta labor de detección en el aula, presentamos un protocolo de observación sistemática en el que se muestran los síntomas más comunes que describen los tres principales trastornos de conducta (TDAH, TND y TD). Esta herramienta incluye una escala de valoración tipo Likert con cuatro opciones de respuesta de menor a mayor frecuencia (Nunca, Algunas veces, Con frecuencia, Siempre), las cuales permitirán conocer al docente el posible trastorno manifiesto por el niño/a o adolescente en función del trastorno que más síntomas presente. Esta metodología observacional, requerirá de una previa formación del profesorado en el campo teórico de los trastornos de conducta, como el que aquí hemos presentado, de tal forma que la cumplimentación del registro se haga con profesionalidad y objetividad. La utilización de la metodología observacional «propone la cuantificación del comportamiento espontáneo que ocurre en situaciones no preparadas, implicando para su consecución el cumplimiento de una serie ordenada de etapas. Su finalidad se materializa en la resolución de problemas (...) planteados acerca de la conducta manifiesta de sujetos que se hallan en un ámbito natural» (Anguera, 1990, 126).

Este procedimiento por parte del docente, servirá como detección e identificación primaria en el aula, la cual deberá ser corroborada en el tiempo con sucesivas cumplimentaciones (por el criterio diagnóstico que implica la permanencia en el tiempo de los trastornos de conducta). A ello sumar, las diversas pruebas y un diagnóstico psicopedagógico más austero por parte de los Servicios de Orientación del Centro y demás agentes especializados del ámbito sanitario. De esta forma, la información recogida de las diversas fuentes y observadores, permitirá realizar una evaluación interdisciplinar del menor para establecer el tratamiento más adecuado a sus necesidades o particularidades. 


\begin{tabular}{|l|l|l|l|l|l|}
\hline $\begin{array}{l}\text { PROTOCOLO DE OBSERVACIÓN DE } \\
\text { LOS TRASTORNOS CONDUCTUA- } \\
\text { LES EN EL AULA }\end{array}$ & \multicolumn{4}{|c|}{ ESCALA DE VALORACIÓN } \\
\hline $\begin{array}{l}\text { Alumno/a: } \\
\text { Observador/a: }\end{array}$ & $\begin{array}{l}\text { Edad: } \\
\text { Fecha: }\end{array}$ & Nunca & $\begin{array}{c}\text { Algunas } \\
\text { Veces }\end{array}$ & $\begin{array}{c}\text { Con } \\
\text { Frecuencia }\end{array}$ & Siempre \\
\hline \hline
\end{tabular}

TRASTORNO POR DÉFICIT DE ATENCIÓN, HIPERACTIVIDAD E IMPULSIVIDAD

\section{INATENCIÓN}

No presta atención a los detalles

Tiene dificultad para mantener la concentración (en el tiempo previsto para la actividad)

Se distrae con facilidad ante un estímulo externo

Está en las nubes como «ensimismado» en sus pensamientos

Parece que no escucha cuando se le habla

Presenta dificultad para acabar las tareas empezadas

Presenta dificultad para seguir instrucciones u obligaciones

Es olvidadizo

Es desordenado y le cuesta organizar sus tareas

Tiene dificultades en el aprendizaje escolar

Rehúye realizar tareas que supongan mantener la atención

HIPERACTIVIDAD

Se mueve continuamente de su asiento

No permanece sentado cuando así lo exige la tarea o situación 


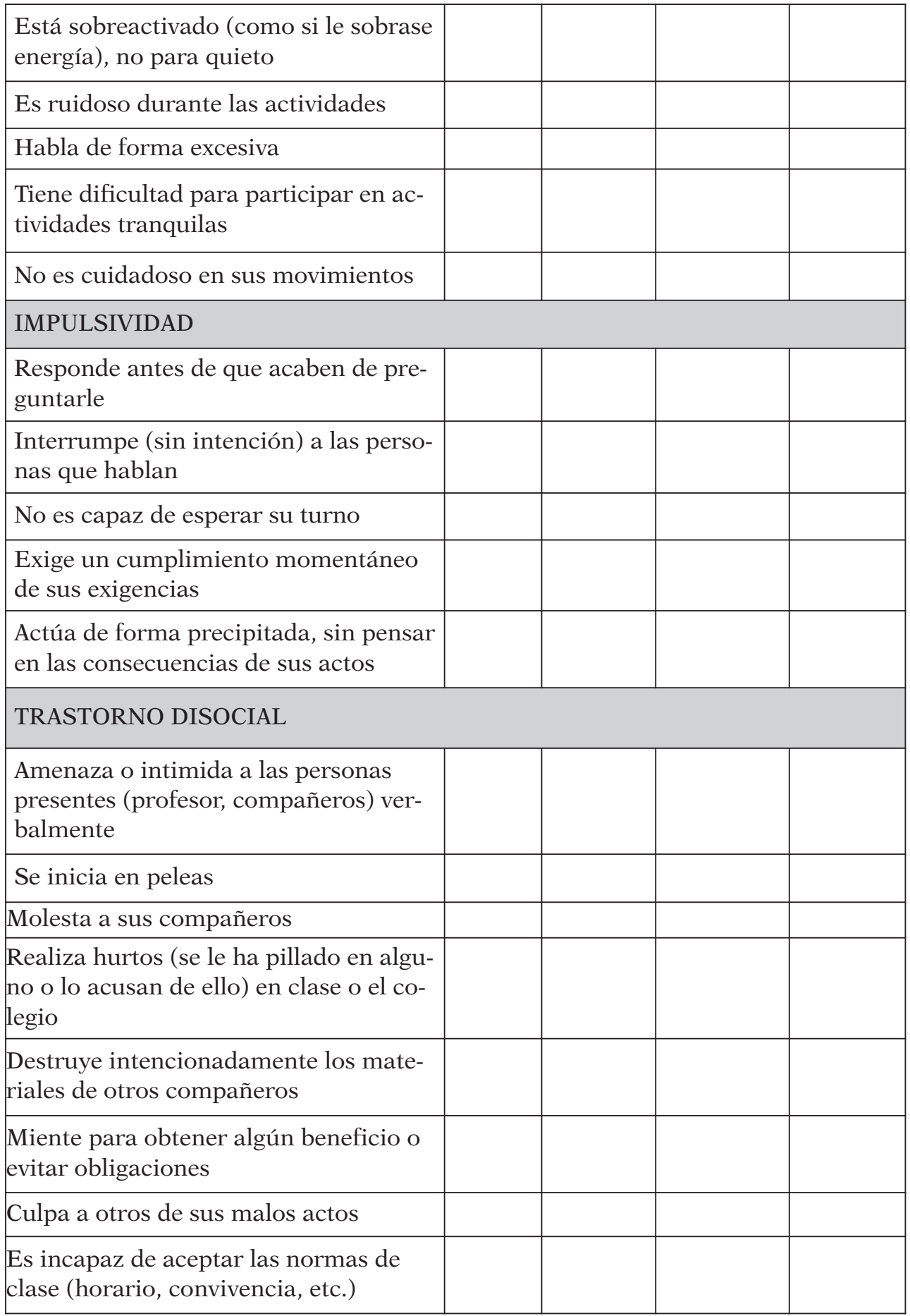




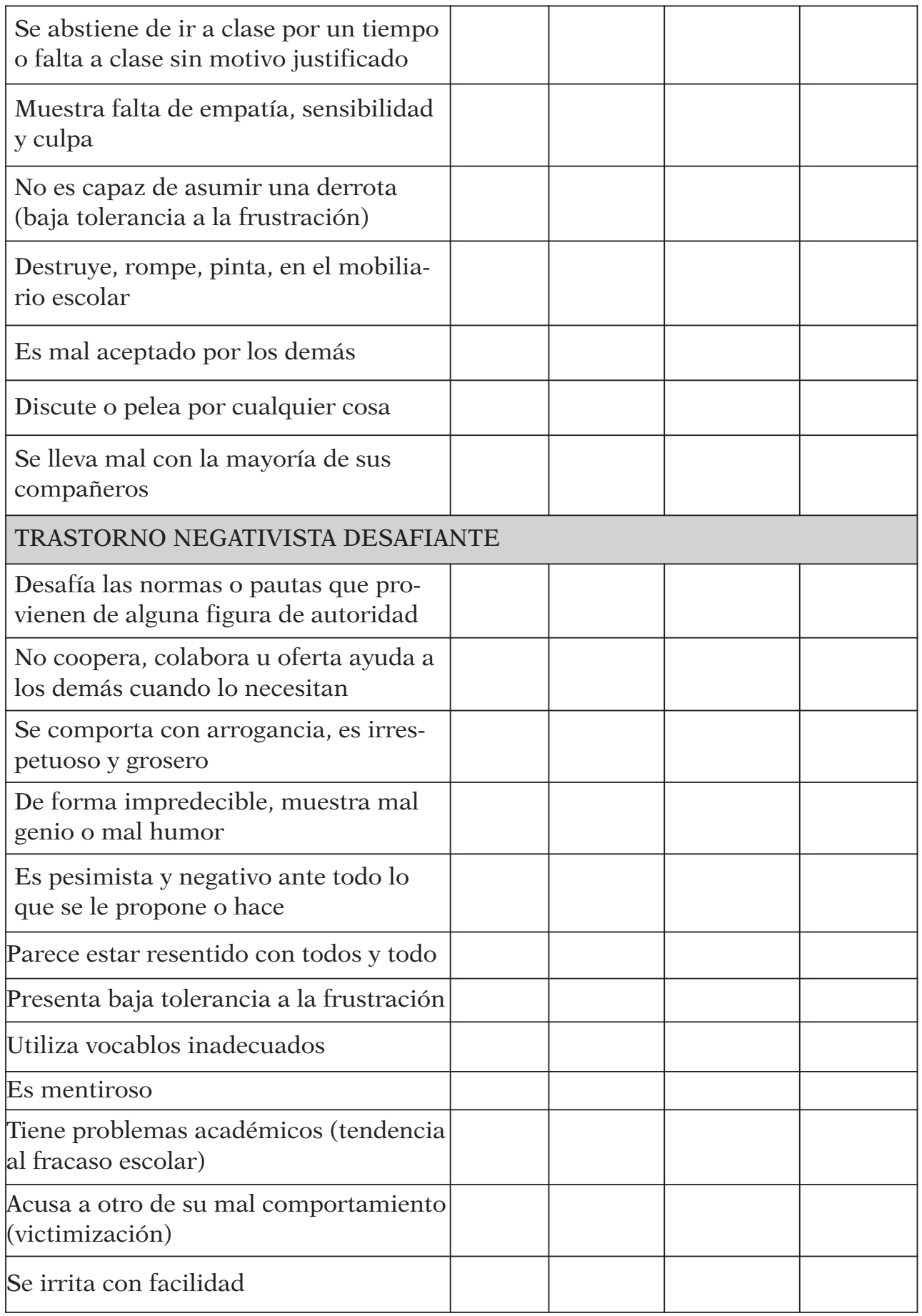


OTRAS OBSERVACIONES A CONSIDERAR:

\section{PROPUESTAS DE INTERVENCIÓN TERAPÉUTICA EN EL AULA}

Tras haber presentado la propuesta de instrumento observacional para la detección de alumnado con trastorno de la conducta en el aula educativa, cabe señalar que, detrás de este trastorno, existe un niño o un adolescente con una historia personal y unas características determinadas. Por ello, nuestro objetivo no será tratar tanto el trastorno como el alumno, siendo ésta la mejor manera de comprenderlos y tratarlos en su individualidad y de una forma holística. La observación diagnóstica a partir del uso del protocolo de observación, acercará al docente y al resto de profesionales implicados, al diseño del posible programa de intervención para la eliminación de conductas desajustadas, o al menos, minimizarlas en la medida de lo posible.

Entendemos junto con Moreno $(2001,169)$ que «la intuición personal, la experiencia y los conocimientos adquiridos, entre otros medios - como el mismo trabajo continuado con niños y adolescentes en las aulas - le otorgan al profesional de la educación lo que podríamos denominar «capacidad de base» para percibir más allá de la constatación evidente que se da en muchos casos, que algunos de sus alumnos tiene dificultades que no pueden ser solventadas sin una ayuda específica y especial».

Si la herramienta de observación sistemática presentada tiene la finalidad de ayudar a la realización de un cribado por parte del docente, en este apartado finalizamos aportando para cada uno de los trastornos de la conducta revisados, una serie de recomendaciones globales para la intervención correctora con estos niños en el aula, partiendo siempre de las características específicas de cada individuo.

\section{Trastorno por Déficit de Atención e Hiperactividad (TDAH)}

Como inicio, consideramos preciso flexibilizar la organización y el funcionamiento de los centros según las necesidades educativas que presenta la diversidad de alumnado escolarizado, a la vez que proporcionar ambientes estructurados que posibiliten y ayuden a la mejora del aprendizaje. De forma específica, algunas de las medidas de intervención a considerar para este trastorno por parte del docente, podrían ser: 
- Establecer prioridades dependiendo de la sintomatología y déficits percibidos.

- Diseñar un programa de trabajo individual basado en los problemas, sobre el escenario emocional y el estilo de aprendizaje del niño. Para ello deberá incluir un autoanálisis sobre su estilo de enseñanza, intentando ante todo ser organizado, emocional y carismático.

- Proporcionar al alumno instrucciones individuales breves, específicas y paso a paso.

- Dosificar la cantidad de información ofertada al alumno, distribuyendo la tarea/s en diferentes partes y destacando la información importante en la pizarra o verbalmente.

- Reducir la cantidad de tarea que se le asigna al niño (para lograr que mantenga la atención en esos periodos cortos de tiempo).

- Negociar el tiempo de trabajo con el niño.

- Otorgar un tiempo extra para completar el trabajo, aunque con limitación.

- Animar al niño a emplear listas de trabajo (tarea por tarea) y a usar organizadores diarios. Todo ello deberá quedar informatizado para el seguimiento de aprendizaje y conductual del menor.

- Reestructurar el escenario ambiental alejando al menor de distractores tales como puertas, ventanas, últimas filas, áreas de ruido y otros escolares ruidosos y diseñando una zona tranquila del aula para trabajar.

- Lograr que el niño comprenda la tarea pues de esta forma el individuo se sentirá motivado para realizar la tarea.

- Animar al alumno a que termine una tarea por sí mismo empezando por pequeños trabajos.

- Hacer consciente al alumno de sus éxitos, logrando mantener su autoestima alta. "La autoestima es un objetivo educacional» (Vaquerizo, Estévez y Díaz, 2006).

\section{Trastorno Negativista Desafiante (TND) y Trastorno Disocial (TD)}

Tras la verificación por parte del docente de la existencia de este tras- 
torno, es necesaria la participación de un equipo multiprofesional. La intervención terapéutica del profesor no va a ser suficiente aunque sí imprescindible, para eliminar o minimizar las conductas disruptivas del alumno. Así, la detección temprana y su posterior derivación a los Equipos de Orientación y a los centros de referencia de salud mental, sería la parte fundamental a cumplimentar por el docente dentro de este desorden.

Una vez que el menor ha sido derivado, el primer paso para iniciar el programa de intervención, ineludiblemente es la evaluación psicopedagógica. En ambos trastornos, las medidas a implementar en el aula por parte del docente, vienen a mitigar estas conductas disruptivas y sirven como complemento ineludible a los tratamientos indicados por el resto de profesionales implicados en la resolución del trastorno. Algunas pautas de actuación para niños y adolescentes con TND y TD, serían:

- Aplicar el manual de convivencia, en aras de que los alumnos comprendan la necesidad de cumplir las normas establecidas. Sería bueno la creación de una serie de normas de conducta de aula claras y explícitas que implique a estos niños como protagonistas en su redacción, sintiéndose partícipes y responsables en su cumplimiento.

- Hacer entender al alumno que con estas conductas provocan daño y el enfado de los demás. Para ello se realizarán dinámicas de grupo que trabajen aspectos como la autoestima y la empatía colectiva.

- No dejarse manipular.

- Intentar que no nos afecte en el comportamiento para con ellos.

- Tener siempre la misma respuesta sin depender de nuestro estado de ánimo.

- Facilitar la posibilidad de elección y desarrollo de las tareas establecidas.

- Desarrollar una planificación realista del trabajo, evitando sobrecargar al alumnado con tareas lejanas a sus intereses y fomentando el trabajo cooperativo.

- Corregir al alumno de forma respetable delante de sus compañeros. Generar algún tipo de rechazo o vergüenza ajena, hará que el menor se revele.

- Trabajar siempre en colaboración con los padres. 
- Reconocer los errores cometidos, demostrando honradez ante el alumnado.

- Graduar las tareas escolares en función de la dificultad, intentando siempre que sean motivadoras y atrayentes para los alumnos.

- Enseñar técnicas de estudio y de aprendizaje a partir de mapas conceptuales, diagramas, etc., que le ayude a ver que aprenden y la importancia de ello.

- Establecer refuerzos positivos.

- Enseñar a los alumnos la importancia de escuchar para aprender.

- No prestar atención ante faltas leves.

- Utilizar silencios y latencias a modo de llamadas de atención y respeto ante la intervención.

- Mostrar un acercamiento y no rechazo al alumno que se muestra más conflictivo.

- Utilizar el humor para minimizar las disrupciones (siempre y cuando no existan faltas de respeto hacia el docente o el resto de compañeros).

- Hacer uso de mensajes en primera persona para que vean la implicación personal en lo que se está transmitiendo.

- Establecer junto con los alumnos castigos hacia sí mismo, ante conductas perturbadoras.

- Trabajar la reflexión e implicación grupal ante diversas situaciones.

- Realizar reuniones de clase para tratar diversos temas que les interesen.

- Hacer uso de la tecnología, ya que los estudiantes con estos desórdenes suelen ser hábiles con los ordenadores y programas activos.

- Ante alumnos que interrumpen de forma constante en el aula:

- Intentar hablar a solas con ellos/ellas 
- Hacerles conscientes de los sentimientos y sensaciones que despiertan en el resto de sus compañeros las constantes interrupciones.

- Procurar establecer algún acuerdo de conducta en clase.

- Ante alumnos agresivos:

- Disminuir la frecuencia o intensidad del comportamiento a través de la enseñanza en técnicas de autocontrol y resolución de conflictos.

- Plantarles cara sin provocar la agresividad evitando luchas de poder.

- Mantener el control visual.

— No dar muestras de enfado ni de debilidad.

- No consentir de ninguna forma la agresividad contra otras personas.

- Aislarlo para que no tenga público.

- Expulsión del aula (en los casos en que pierda el autocontrol).

- Intentar comprender lo que les preocupa cuando se hayan tranquilizado.

- Poner fechas límites para su actitud.

- Hacerles comentarios positivos.

- No discutir nunca con ellos/as.

- Intentar que busquen soluciones a sus propias actitudes (autoresponsabilidad) y reflexionen sobre las consecuencias de sus acciones.

- Evitar darles la razón (cuando no la tengan).

- Parafrasear.

— Interrumpirle lo más pronto posible.

- Hacer preguntas para que reflexionen sobre sus lamentos. 
- Entrevistarlos personalmente y entrevistar a la familia para conocer su comportamiento fuera del aula.

- Ante alumnos con dificultad de relación social:

— Intentar iniciar conversaciones sobre cosas intranscendentes.

- Utilizar siempre preguntas directas.

- Utilizar el Lenguaje corporal amigable.

- No terminar nunca las respuestas aunque sean muy lentas.

- Promover el trabajo cooperativo con sus compañeros.

A modo de conclusión, puesto que los trastornos de la conducta se están convirtiendo en uno de los motivos de consulta clínica más frecuentes durante la infancia y adolescencia, «el papel de los profesores y de los orientadores de los colegios y centros educativos es fundamental para la detección precoz y el diagnóstico» (Mardomingo, 2004). El acercamiento multidisciplinar a esta problemática es una pieza clave para no sólo detectar estos casos entre nuestros estudiantes, sino para asegurar la derivación a profesionales especializados de forma temprana, evitando consecuencias futuras negativas como el trastorno de personalidad en la vida adulta.

Una detección precoz y un abordaje terapéutico adecuado por los distintos profesionales inmersos en el proceso educativo puede ser un punto de inflexión en la prevalencia de estas patologías en el aula y demás contextos. Si aportamos a los docentes y Equipos de Orientación una formación básica de calidad, los medios adecuados y un correcto flujo comunicativo entre la escuela, la sanidad, la familia (o servicios sociales, en su caso) podremos eliminar la curva de tendencia positiva con respecto a la aparición y mantenimiento de alumnos con trastorno del comportamiento. 


\section{REFERENCIAS BIBLIOGRÁFICAS}

Alonso, J. y Navazo, M. A. (2002). La agresividad escolar y su relación con el rendimiento escolar. Revista Electrónica Interuniversitaria de Formación del Profesorado, 5 (1), 1-5.

American Academy of Child and Adolescent Psychiatry -AACAP- (1997). Practice parameters for the assessment and treatment of children and adolescents with conduct disorder. Journal of the American Academy of Child and adolescent Psychiatry, 36 (10), 122-139.

American Psychiatric Association (2002). Manual Diagnóstico y Estadístico de los Trastornos Mentales. DSM-IV-TR. Barcelona: Masson.

Anguera, M. T. (1990). Metodología Observacional, en Arnau, J.; Anguera, M. T. y Gómez, J. Metodología de la investigación en ciencias del comportamiento. Murcia: Servicio de Publicaciones de de Murcia, 125-236.

Angulo, M. C.; Fernández, C.; García, F. J.; Giménez, A. M.; Ongallo, C. M.; Prieto, I. y Rueda, S. (2008). Manual de atención al alumnado con necesidades específicas de apoyo educativo derivadas de trastornos graves de conducta. Andalucía: Junta de Andalucía, Consejería de Educación y Dirección General de Participación e Innovación Educativa.

Artigas, J. (2003). Comorbilidad en el trastorno por déficit de atención/hiperactividad. Revista de Neurología, 36 (1), 68-78.

Atienza, J. M. (n.d.). Prevalencia de los trastornos de conducta [en línea]. Disponible en: http://www.paidopsiquiatria.com/TDAH/tc7.pdf [consulta: 2011, 12 de Marzo].
Bierderman, J.; Mick, E.; Faraone, S. V.; Braaten, E.; Doyle, A.; Spencer, T.; Wilens, T. E.; Frazier, E. y Johnson, M. A. (2002). Influence of gender on Attention Deficit Hyperactivity Disorder in children referred to a psychiatric clinic. American Journal of Psychiatry, 159, 36-42.

Domínguez, J. y Pino, M. R. (2008). Las conductas problemáticas en el aula: propuesta de actuación. Revista Complutense de Educación, 19 (2), 447-457.

Félix, V. (2007). Conceptualización del comportamiento disruptivo en niños y adolescentes [en línea]. Disponible en: http://www.uv.es/femavi/Elda2.pdf [consulta: 2011, 3 de Marzo].

Gerard, R. (2002). La agresividad [en línea]. Disponible en: http://www.apsique.com/wiki/AnorAgresividad2 [consulta: 2011, 3 de Marzo].

Greciano, I. (2001). Alteraciones del comportamiento en el aula [en línea]. Disponible en:http://www.eskolabakegune.euskadi.net/c/document_library/get_ file ?uid =a 1 a 1 b8cb-8317-4fcf-a4925e4656def19f\&groupId=2546067 [consulta: 2011, 13 de Marzo].

Hill, J. (2003). Early identification of individuals at risk for antisocial personality disorder. The British Journal of Psychiatry, 182, 11-14.

Jara, A. B. (2009). El TDAH, Trastorno por Déficit de Atención con Hiperactividad, en las clasificaciones diagnósticas actuales (C.I.E. 10, D.S.M.IV-R Y C.F.T.M.E.A.-R 2000). NORTE de Salud Mental, 35, 30-40.

Justicia, F.; Benítez, J. L.; Pichardo, M. C.; Fernández, E.; García, T. y Fernández, M. (2006). Aproximación a un nuevo modelo explicativo del compor- 
tamiento antisocial. Revista Electrónica de Investigación Psicoeducativa, 4 (9), 131-150.

Mardomingo, M. J. (2004). Detección y diagnóstico del alumnado con problemas de conducta [en línea]. Disponible en: http://www.redes-cepalcala.org/inspector/DOCUMENTOS $\% 20 Y \% 2$ 0LIBROS/DERECHOS-DEBERESCONVIVENCIA/DETECCION\%20Y\%2 0 DIAG N O T I C O $\% 20$ P R OBLE MAS\%20CONDUCTA.pdf [consulta: 2011, 20 de Marzo].

Miranda, A.; Jarque, S. y Soriano, M. (1999). Trastorno de hiperactividad con déficit de atención: polémicas actuales acerca de su definición, epidemiología, bases etiológicas y aproximaciones a la intervención. Revista de Neurología, 28 (2), 182-188.

Montiel, C. y Peña, J. A. (2001). Discrepancia entre padres y profesores en la evaluación de problemas de conducta y académicos en niños y adolescentes. Revista de Neurología, 32 (6), 506-511.

Moreno, F. X. (2000). Diagnóstico de los Factores de Riesgo como Recurso Preventivo de los Problemas de Conducta en el Contexto Escolar. Revista Electrónica Convenid II, 29-44.

Moreno, F. X. (2001). Análisis psicopedagógico de los alumnos de Educación Secundaria Obligatoria con problemas de comportamiento en el contexto escolar. Tesis Doctoral inédita, Departament de Psicologia de la salud i Psicologia Social, Universitat Autònoma de Barcelona.

Obrero, O. (2009). Los trastornos disociales en el aula. Revista Digital Enfoques Educativos, 37, 86-132.

Oldham, J. M.; Skodol, A. E. y Bender, D. S. (2007). Tratado de los trastornos de la personalidad. Barcelona: MASSON.
Organización Mundial de la salud (2008). Guía de bolsillo de la Clasificación CIE-10. Madrid: Médica Panamericana.

Palomero, J. E. y Fernández, M. R. (2001). La violencia escolar: un punto de vista global. Revista Interuniversitaria de Formación del Profesorado, 41, 19-38.

Peña, J. A. y Montiel, C. (2003). Trastorno por déficit de atención/hiperactividad: ¿mito o realidad? Revista de Neurolología, 36 (2), 173-179.

Raya, A. F.; Pino, M. J. y Herruezo, J. (2009). La agresividad en la infancia: el estilo de crianza parental como facto relacionado. European Journal of Educational and Psychology, 2 (3), 211222.

San Sebastián, J.; Soutullo, C. y Figueroa, A. (2010). Trastorno por Déficit de Atención e Hiperactividad (TDAH), en Soutullo, C. y Mardomingo, M. J. Manual de Psiquiatría del Niño y del Adolescente. Madrid: Médica Panamericana, 55-78.

Sibon, A. M. (2010). Los trastornos de conducta en el aula. Revista Digital Innovación y Experiencias Educativas, $28,1-11$.

Soler, M. (2010). Problemas de comportamiento y técnicas de modificación de conducta. Revista Digital Innovación y Experiencias Educativas, 35 [en línea] Disponible en: http://www.csicsif.es/andalucia/modules/mod_ense/re vista/pdf/Numero_35/MIRIAM\%20SOLER\%20PENA_1.pdf [consulta: 2011, 12 de Marzo].

Steiner, H. y Remsing, L. (2007). Practice parameter for the assessment and treatment of children and adolescents with oppositional defiant disorder. Journal of the American Academy of 
Child and Adolescent Psychiatry, 46 (1), $\quad$ intervención psicolingüística en el tras126-141.

torno por déficit de atención e hipe-

Vaquerizo, J.; Estévez, F. y Díaz, I.

(2006). Revisión del modelo de alerta e ractividad. Revista de Neurología, 42 (2), 53-61. 


\title{
PALABRAS CLAVE
}

Trastorno de la conducta, trastorno por déficit de atención e hiperactividad, trastorno negativista desafiante, trastorno disocial/antisocial, registro observacional, intervención terapéutica.

\section{KEY WORDS}

Behaviour disorder, attention deficit hyperactivity disorder, oppositional defiant disorder, antisocial behaviour, observational tool, therapeutic intervention.

\section{PERFIL ACADÉMICO Y PROFESIONAL DEL AUTOR/ES}

José Antonio Rabadán Rubio, Profesor Contratado Doctor en la Facultad de Educación de la Universidad de Murcia. Master en Dirección de Personal y Gestión de RR.HH., Master en Psicopedagogía Clínica y Especialista en Dirección de Centros Sociales (Comunidad de Madrid). Psicopedagogo clínico en el Hospital Virgen de de Murcia y Psicoclínica.

Líneas de investigación: Trastornos de la conducta infanto-juvenil, inmigración, educación para la salud e intervención socioeducativa.

Ana María Giménez Gualdo, Becaria de Investigación FPU del Ministerio de Educación. Diplomada en Educación Social y Licenciada en Pedagogía. Premio extraordinario fin de carrera en Educación Social, Tercer Premio Nacional al Rendimiento Académico en Educación Social y Premio extraordinario fin de carrera en Pedagogía. Master en Investigación e Innovación en Educación Infantil y Educación Primaria.

Líneas de investigación: Trastornos de conducta, Violencia Escolar (Bullying) y Ciberbullying, Atención a la Diversidad.

\author{
Dirección de los Autores: Prof. D. José Antonio Rabadán Rubio \\ Departamento de Teoría e Historia de la \\ Educación, despacho 1.21 \\ Facultad de Educación. Universidad de Murcia \\ 30100 Campus de Espinardo \\ Ana María Giménez Gualdo \\ Departamento de Didáctica y Organización \\ Escolar, despacho 11B Facultad de Educación \\ Universidad de Murcia \\ 30100 Campus de Espinardo
}


Fecha Recepción del Artículo: 07. Noviembre. 2010

Fecha Revisión del Artículo: 09. Mayo. 2011

Fecha Aceptación del Artículo: 15. Junio. 2011

Fecha de Revisión para publicación. 24. Noviembre. 2011 